\title{
What can Fermi tell us about magnetars?
}

\author{
H. Tong ${ }^{1}$ and R. X. Xu ${ }^{2}$ \\ ${ }^{1}$ Xinjiang Astronomical Observatory, Chinese Academy of Sciences, Urumqi, \\ Xinjiang 830011, China \\ email: tonghao@xao.ac.cn \\ ${ }^{2}$ School of Physics and State Key Laboratory of Nuclear Physics and Technology, \\ Peking University, Beijing 100871, China \\ email: r.x.xu@pku.edu.cn
}

\begin{abstract}
We have analyzed the physical implications of Fermi observations of magnetars. Observationally, no significant detection is reported in Fermi observations of all magnetars. Then there are conflicts between outer gap model in the case of magnetars and Fermi observations. One possible explanation is that magnetars are wind braking instead of magnetic dipole braking. In the wind braking scenario, magnetars are neutron stars with strong multipole field. A strong dipole field is no longer required. A magnetism-powered pulsar wind nebula and a braking index smaller than three are the two predictions of wind braking of magnetars. Future deeper Fermi observations will help us make clear whether they are wind braking or magnetic dipole braking. It will also help us to distinguish between the magnetar model and the accretion model for AXPs and SGRs.
\end{abstract}

Keywords. pulsars: general, stars: magnetars, stars: neutron

\section{Introduction}

Anomalous X-ray pulsars (AXPs) and soft gamma-ray repeaters (SGRs) are peculiar pulsar-like objects. They are commonly assumed to be magnetars, magnetism-powered neutron stars. The traditional model of magnetars is that they are young neutron stars with both strong dipole field and strong multipole field (Duncan \& Thompson 1992; Thompson et al. 2002). The presence of strong dipole field ensures that they can also accelerate particles to very high energy via the outer gap mechanism. Therefore, magnetar may emit high-energy gamma-rays detectable by Fermi-LAT (Cheng \& Zhang 2001). These high-energy gamma-rays are rotation-powered in nature (Zhang 2003). Detection of rotation-powered activities in magnetars will help bridge the gap between magnetars and normal pulsars.

Fermi-LAT has observed the whole magnetar class. No significant detection is reported (Sasmaz Mus \& Gogus 2010; Abdo et al. 2010). Thus there is a conflict between the outer gap model in the case of magnetars and Fermi observations (Tong, Song \& Xu 2010, 2011). Below we will show what Fermi can tell us about magnetars.

\section{Implications of Fermi observations of magnetars}

We have analyzed the implications of the Fermi observation of AXP 4U 0142+61 (Sasmaz Mus \& Gogus 2010; Tong, Song \& Xu 2010). It is shown that there are conflicts between outer gap model in the case of AXP 4 U $0142+61$ and Fermi observations. The fallback disk model for AXPs and SGRs can still not be ruled out. In Fermi observations of the whole magnetar class, still no significant detection is reported. This is consistent with our previous analysis (Abdo et al. 2010; Tong, Song \& Xu 2011). The upper limit of 
AXP 4U 0142+62 lies already below the theoretical calculations for some parameter space (Tong, Song \& Xu 2011). Future deeper Fermi observations will help us to distinguish between the magnetar model and the accretion model for AXPs and SGRs.

\section{Solutions and predictions}

There are two possible explanations to the non-detection in Fermi observations of magnetars. One possibility is that AXPs and SGRs are accretion-powered systems. Then it is natural that they are not high-energy gamma-ray emitters. Various observations of AXPs and SGRs can be explained naturally in the accretion scenario (Tong \& Xu 2011).

The other possibility is that magnetars are wind braking. If magnetars are wind braking instead of magnetic dipole braking, then their magnetosphere structure is different from that of normal pulsars. Vacuum gaps (including outer gap) may not exist in magnetars. This may explain the non-detection in Fermi observations of magnetars (see section 4.2 in Tong et al. 2012).

In the wind braking scenario, magnetars are neutron stars with strong multipole field. A strong dipole field is no longer required. Figure 1 shows the correspond dipole field in the case of wind braking and that of magnetic dipole braking

Recent challenging observations of magnetars may be explained naturally in the wind braking scenario: (1) The supernova energies of magnetars are of normal value; (2) The

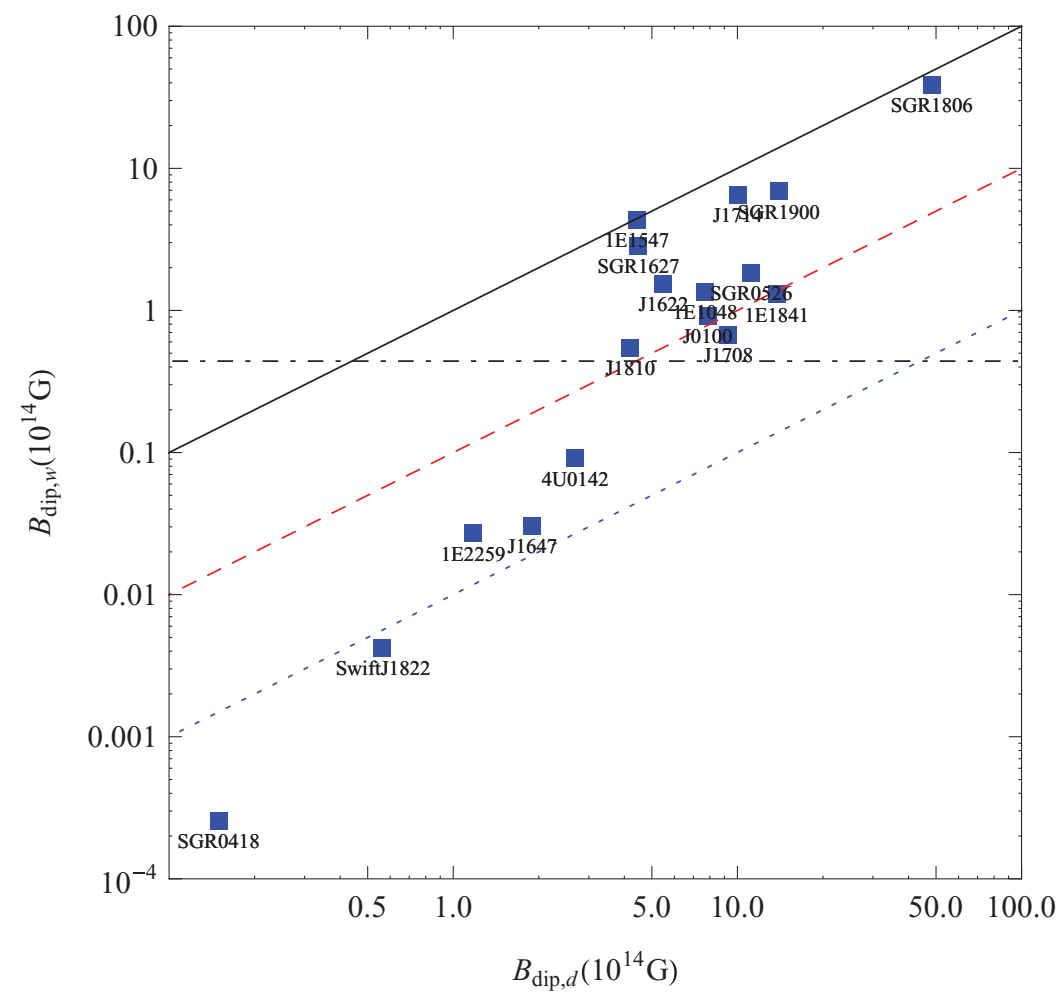

Figure 1. Dipole magnetic field in the case of wind braking versus that in the case of magnetic dipole braking. A wind luminosity $L_{\mathrm{p}}=10^{35} \mathrm{erg} \mathrm{s}^{-1}$ is assumed. The solid, dashed, and dotted lines are for $B_{\mathrm{dip}, \mathrm{w}}=B_{\mathrm{dip}, \mathrm{d}}, 0.1 B_{\mathrm{dip}, \mathrm{d}}, 0.01 B_{\mathrm{dip}, \mathrm{d}}$, respectively. The dot-dashed line marks the position of quantum critical magnetic field $B_{\mathrm{QED}}=4.4 \times 10^{13} \mathrm{G}$. See Figure 2 and corresponding text in Tong et al. (2012) for details. 
problem posed by the low-magnetic field soft gamma-ray repeater; (3) The relation between magnetars and high magnetic field pulsars; and (4) A decreasing period derivative during magnetar outbursts. A magnetism-powered (instead of rotation-powered) pulsar wind nebula will be one of the consequences of wind braking. For a magnetism-powered pulsar wind nebula, we should see a correlation between the nebula luminosity and the magnetar luminosity. The extended emission around AXP 1E 1547.0-5408 may be a magnetism-powered pulsar wind nebula. Under the wind braking scenario, a braking index smaller than three is expected. More details are presented in Tong et al. (2012).

Considering that magnetars are wind braking (both a rotation-powered and a magnetism-powered particle wind), many aspects of magnetars can be reinterpreted. For example, the "low magnetic field" magnetar SGR 0418+5729 may actually be a normal magnetar (Rea et al. 2010; Tong \& Xu 2012). It is a little special since it has a special geometry, e.g. a small magnetic inclination angle. Another example is that low luminosity magnetars are more likely to have radio emissions. The reason is that low luminosity magnetars may have a similar magnetospheric structure as normal radio pulsars (Rea et al. 2012; Liu, Tong \& Yuan 2012).

\section{Conclusions}

Fermi observations of magnetars suggest magnetars may be wind braking instead of magnetic dipole braking. Future deeper Fermi-LAT observations will help us make clear whether magnetars are wind braking or magnetic dipole braking. It will also help us to distinguish between the magnetar model and the accretion model for AXPs and SGRs. Therefore, deeper Fermi-LAT observations of the magnetar class in the future are highly recommended.

\section{Acknowledgments}

This work is supported by the National Basic Research Program of China (2009CB824800), the National Natural Science Foundation of China (11103021, 10935001, 10973002), and the John Templeton Foundation.

\section{References}

Abdo, A. A., Ackermann, M., Ajello, M., et al. 2010, ApJ, 725, L73

Cheng, K. S. \& Zhang, L. 2001, ApJ, 562, 918

Duncan, R. C. \& Thompson, C. 1992, ApJ, 392, L9

Liu, Z. Y., Tong, H., \& Yuan, J. P. 2012, arXiv:1210.2799

Rea, N., Esposito, P., Turolla, R., et al. 2010, Science, 330, 944

Rea, N., Pons, J. A., Torres, D. F., et al. 2012, ApJ, 748, L12

Sasmaz Mus, S. \& Gogus, E. 2010, ApJ, 723, 100

Thompson, C., Lyutikov, M., \& Kulkarni, S. R. 2002, ApJ, 574, 332

Tong, H., Song, L. M., \& Xu, R. X. 2010, ApJ, 725, L196

Tong, H., Song L. M. \& Xu, R. X. 2011, ApJ, 738, 31

Tong, H. \& Xu, R. X. 2011, Int. Jour. Mod. Phys. E, 20, 15

Tong, H. \& Xu, R. X. 2012, ApJ, 757, L10

Tong, H., Xu, R. X., Song, L. M., \& Qiao, G. J. 2012, arXiv:1205.1626

Zhang, B. 2003, Astrophysics and Space Science Library, 298, 27 (astro-ph/0212016) 